\title{
Pain perception and laser evoked potentials during menstrual cycle in migraine
}

\author{
Marina de Tommaso - Massimiliano Valeriani - Michele Sardaro • \\ Claudia Serpino · Olimpia Di Fruscolo · Eleonora Vecchio · \\ Rosanna Cerbo · Paolo Livrea
}

Received: 14 April 2009/Accepted: 6 August 2009/Published online: 10 September 2009

(C) Springer-Verlag 2009

\begin{abstract}
The association between estrogens "withdrawal" and attacks of migraine without aura is well-known. The aim of the study was to examine the features of laser evoked potentials (LEPs), including habituation, in women suffering from migraine without aura versus healthy controls, during the pre-menstrual and late luteal phases. Nine migraine without aura and 10 non-migraine healthy women, were evaluated during the pre-menstrual phase and late luteal phase. The LEPs were recorded during the inter-critical phase. The right supraorbital zone and the dorsum of the right hand were stimulated. Three consecutive series of 20 laser stimuli were obtained for each stimulation site. Laser pain perception was rated by a $0-100$ VAS after each stimulation series. Migraine patients exhibited increased LEPs amplitude and reduced habituation compared to normal subjects. Laser-pain perception was increased during the pre-menstrual phase in both patients and controls. Migraine patients and controls showed increased P2 and N2-P2 amplitude in the pre-menstrual phase, on both stimulation sites. During the pre-menstrual phase the N2-P2 habituation appeared to be reduced in both migraine and healthy women. The
\end{abstract}

M. de Tommaso · M. Sardaro · C. Serpino ·

O. D. Fruscolo $\cdot$ E. Vecchio $\cdot$ P. Livrea

Neurophysiopathology of Pain Unit, Neurological and

Psychiatric Sciences Department, University of Bari, Bari, Italy

M. Valeriani

Bambin Gesù Hospital, Rome, Italy

R. Cerbo

Pain Centre, Neuroscience Department,

La Sapienza University, Rome, Italy

M. de Tommaso $(\square)$

"Amaducci" Neurological Clinic, Policlinico General Hospital, Piazza Giulio Cesare 11, Bari 70124, Italy

e-mail: m.detommaso@neurol.uniba.it estrogen withdrawal occurring during the menstrual cycle may favor reduced habituation of nociceptive cortex, which may facilitate pain symptoms and migraine in predisposed women.

Keywords Migraine $\cdot$ Menstrual cycle $\cdot$ Pain sensitivity . Laser evoked potentials

\section{Introduction}

Women suffer a disproportionate amount of pain during their lifetime compared to men. Over the past 15 years, a growing number of studies have suggested several causes for this sex difference, ranging from cellular to psychosocial levels of analysis [1]. Migraine, an incapacitating disorder based on altered neuronal excitability [2-4], is three times more common in women during reproductive years than in men of a similar age [5]. Initial evidence for the role of estrogen in migraine headache stems from studies, published in the early 1970s [6], which evaluated the influence of exogenous estrogens on women prone to migraine around the time of menses. Indeed, migraine attacks were correlated with declining plasma estradiol levels [6]. This was particularly evident after prolonged estradiol elevations, as those observed before menses [7-9]. However, estrogenic modulation of pain per se is a complex phenomenon, since estrogens may have either a pro- or an anti-nociceptive effect [1]. There is neurophysiologic evidence about the role of menstrual cycle in increasing the probability of migraine attacks, based on the amplitude changes of an event-related potentials-the contingent negative variation $(\mathrm{CNV})$, - whose abnormalities have been referred to the neuronal factors predisposing to migraine $[10,11]$. In a recent study about pain sensations to the cold presser test in normally 
menstruating women, pain threshold was found reduced during the late luteal phase compared with the pre-menstrual phase [12]. However, other studies on pain thresholds during the menstrual cycle reported uncertain results in healthy [13] and fibromyalgia suffering women [14].

The majority of evoked and event-related potentials studies in migraine have shown two abnormalities: increased amplitudes of averages of large numbers of trials and lack of habituation in successive trial blocks during the pain-free phase, with the ictal normalization of evoked potential amplitudes and habituation [15]. The evoked potentials amplitude is a quantitative index of the neuronal population activated by certain sensory inputs; it tends to decrease during repetitive sensory stimulation, as the expression of a progressive reduction of the neuronal response. The physiologic phenomenon of a sensory cortex, which progressively reduces its activity in being reached by repetitive stimuli, is known as habituation. According to the definition by Thompson and Spencer [16], the habituation is a phenomenon of decrement in the amplitude of sensory cortical responses to repeated presentations of similar stimuli, excluding receptor or effectors fatigue, in order to avoid brain over-stimulation.

There is growing evidence about the utility of laser evoked potentials (LEPs) in the clinical management of primary headaches [17]. In migraine, during the interictal phase, LEPs have normal amplitudes in basal conditions, but they show a reduced habituation to repetitive stimuli and an altered modulation by attention tasks [18-20]. During the migraine attack, LEPs are enhanced and laserpain perception is increased, suggesting central sensitization phenomena [21], which may subtend the persistence of reduced habituation pattern to repetitive nociceptive stimuli [20]. LEPs may provide an objective measurement of cortical pain processing during the different phases of menstrual cycle in both healthy and migraine women.

The aim of the study was to evaluate pain perception and brain responses to laser stimuli during the late luteal versus pre-menstrual phase in normally menstruating migraine without aura patients and in non-migraine healthy women. In addition, also the LEP habituation to repetitive series of stimulations was investigated.

\section{Methods}

\section{Subjects}

The study design planned the selection of normally menstruating women suffering from migraine without aura among the patients coming to the Neurophysiopathology of Pain Unit of the Neurological and Psychiatric Sciences Department of Bari University. They were compared to normally menstruating non migraine healthy subjects, selected among the medical and technical staff. The diagnosis was made according to the criteria of the International Headache Society (code 1.1) [22] by the first author (neurologist) trained in diagnosis and treatment of headaches. Before being included in the study, all migraineurs and controls were asked to keep a headache diary and a diary of the menstrual cycle for 3 months in order to collect clinical data and, for patients, to assess the relationship between migraine and menstruation. The exclusion criteria were similar for both groups and included the following ones: consumption of central nervous system acting drugs in the last 3 months, history of psychopathological or neurological disorders, major medical problems, and oral contraceptive drugs therapy in the last 3 months.

Migraineurs should not have used prophylactic migraine medication for at least 3 months prior to the investigation, nor triptans or analgesics in the $72 \mathrm{~h}$ preceding the recording session. Migraine patients were examined in the asymptomatic condition, at least $72 \mathrm{~h}$ following the last attack and in the $48 \mathrm{~h}$ preceding the next one. In the course of the study, we observed that this latter criterion was quite impossible to be satisfied in all cases because of the early occurrence of migraine attack after the pre-menstrual phase recording session. Thus, we decided to tolerate an interval $\geq 24 \mathrm{~h}$ following the experimental trial. The Ethics Committee of the Bari Policlinico General Hospital approved the study. The subjects were informed about the course of the experiment and gave written informed consent according to the Helsinki Declaration. Among 20 initially selected migraineurs without aura, only nine satisfied the requested criteria. In fact, six patients were excluded because a migraine attack occurred less than $24 \mathrm{~h}$ after the LEPs recording session, three patients experienced headache in the $72 \mathrm{~h}$ preceding the task, while two patients did not give full cooperation to complete the study. The included patients were all affected by menstrual-related migraine without aura, according to the proposed subclassification of the International headache society [22]. Migraine women were $26 \pm 6.8$ years old, with mean migraine duration of $9.8 \pm 4.1$ years and a mean headache frequency $4 \pm 2.94$ days with headache/month in the last 3 months. We also selected 15 non-migraine normally menstruating healthy subjects, but only 10 completed the study, while five controls were lost to one of the recording session, one for pregnancy, two for non-compliance, two for an occurrence of menstrual cycle later than 2 days after the LEP recording. They were $26.8 \pm 5.3$ years old.

\section{Experimental design}

In each subject, LEP recordings were performed in the premenstrual and late-luteal phases of the menstrual cycle. 
A rise in the basal body temperature (BBT) was used to confirm the ovulation occurrence. For this purpose, patients were given basal thermometers and charts for recording BBT. The onset of the next menses was estimated by information supplied by patients relating to the length of the last three menstrual cycles and the observation of a $0.3-$ $0.5^{\circ} \mathrm{C}$ rise in BBT. In all subjects recordings were carried out $14 \pm 0.74$ days and 1-2 days before the occurrence of menstruation. The mean length of the menstrual cycle was $27.45 \pm 1.1$ days and $27.5 \pm 1.2$ days in the migraine and healthy group, respectively. All the selected patients experienced a migraine attack during the menstruation following the recording session. LEP recording followed the last attack from 72 to $124 \mathrm{~h}(90 \pm 9.1 \mathrm{~h})$; it preceded the next attack ranged from 25 to $50 \mathrm{~h}$ in the pre-menstrual phase (mean $33 \pm 5.7 \mathrm{~h}$ ) and from 50 to $180 \mathrm{~h}$ in the late luteal phase. The occurrences of menstruation, as well as the onset of a migraine attack, were controlled by telephone contact.

\section{Laser evoked potentials}

\section{Stimulation}

Cutaneous heat stimuli were delivered by a $\mathrm{CO}_{2}$ laser (wavelength $10.6 \mu \mathrm{m}, 2 \mathrm{~mm}$ beam diameter-ELEN, Florence, Italy) on the dorsum of the right hand and the right supraorbital zone. We chose to limit the study to a right sided stimulation in order to avoid a lengthy, uncomfortable procedure and because none of the selected migraineurs reported a higher attack prevalence on one side. The stimulation site was visualized by a He-Ne laser beam. The location of the impact on the skin was slightly shifted between two successive stimuli, to avoid the sensitization of the nociceptors. $\mathrm{CO}_{2}$ laser stimuli were delivered at a fixed power of $7.5 \mathrm{~W}$ and duration of $25 \mathrm{~ms}$ [23], which was perceived by all patients and controls as a painful pinprick. We took attention to settle the laser power and duration at a suprathreshold level in all cases [23], using a 10-point verbal analog scale in which ' 0 ' corresponds to no sensation, '4' to the pain threshold and ' 10 ' to intolerable pain. In all patients and controls the $25 \mathrm{~ms}$ duration and $7.5 \mathrm{~W}$ intensity laser stimuli were judged as a painful pinprick, with a value $>6$ in more than $50 \%$ of 20 stimuli.

Patients and controls were examined in the ovulation and pre-menstrual phases, stimulating in random order the hand and facial zone. In order to assess habituation, three consecutive series of 20 laser stimuli were delivered, with and inter-stimulus interval (ISI) in the range of 10-15 s, and an interval between the series of $5 \mathrm{~min}$ [18]. All subjects were requested to take attention to the stimuli and to count them. At the end of each stimulation series, they had also to rate pain induced by laser. In order to assess pain intensity, a 0-100-point VAS, in which '0' corresponds to no pain and ' 100 ' to the worst pain one may conceive, was used.

\section{Recording}

During LEPs recording, the subjects lay on a couch in a warm and semi-dark room; they were awake and relaxed, with eyes open. Both the subject and the experimenters wore protective goggles or glasses during data acquisition. LEPs were obtained using surface recording electrodes, placed at $\mathrm{Cz}$ and $\mathrm{Pz}$, referred to the nasion, and $\mathrm{T} 3$ and $\mathrm{T} 4$ positions, referred to $\mathrm{Fz}$ derivation (10-20 international system), by means of MICROMED EEG apparatus (Micromed Brain Quick, Mogliano Veneto, Italy). A further electrode was positioned above the right eyebrow for electro-oculogram (EOG) recording. The ground electrode was at Fpz.

\section{LEPs analysis}

An investigator blind to the clinical condition and menstrual phase analyzed the LEPs. They were analyzed for 1-s with $100 \mathrm{~ms}$ pre-stimulus time at a sampling rate of $512 \mathrm{~Hz}$, using the advanced source analysis (ASA) vers. 4.6 by ANT software. All runs containing transients exceeding $65 \mu \mathrm{V}$ at each recording channel were excluded from the average by an automatic artifact rejection algorithm. In addition, further artifacts were visually inspected and an average of at least 15 artifact-free responses was obtained off-line, for each stimulation series.

For each stimulation site, an average across the single series of stimuli was obtained for the right hand and right supra-orbital zone. LEPs were identified based on their latency and distribution, and three responses were labeled according to Valeriani et al. [24]. The N2a (namely N2) and $\mathrm{P} 2$ components were analyzed at the vertex $(\mathrm{Cz})$ and the N1 component was analyzed at T3-Fz trace. Absolute latencies of the scalp potentials were measured at the highest peak of each response component and the amplitude of each wave was measured from the baseline. Baseline was measured automatically by calculating the average signal on the whole sweep and subtracting it from the trace (ASA-vers. 4.6 by ANT software). In addition, the peak-to-peak amplitude was taken into consideration for the vertex biphasic LEP component (N2-P2). All the waves were identified in a time window compatible with the mean latencies indicated by Cruccu et al. [25].

Statistical analysis

After the Kolmogorov-Smirnov statistic, with a Lilliefors significance level, was applied for testing normality, the 
mean VAS values and the N1 and N2-P2 amplitudes and latencies, computed across the three repetitions, were the variables for a two-way ANOVA analysis, where the diagnosis and the menstrual phase were the main factors. In order to assess the outcome of LEP habituation in the different phases, we computed the ratio between the N2-P2 amplitudes recorded in the third and in the first stimulation series. The influence of diagnosis and menstrual phase was evaluated, using a two-way ANOVA with diagnosis and phase as factors. The SPSS software, vers. 11 for Windows, was employed.

\section{Results}

\section{LEP latencies}

LEP latencies were not significantly modified in the two phases across the two groups.

\section{LEP amplitudes}

Migraine patients exhibited enhanced N2 and P2 wave amplitudes, with an increase of the N2-P2 complex, at the hand level, and increased P2 and N2-P2 amplitudes, at the trigeminal level, when compared with non migraine women (Fig. 1; Table 1). In both groups, at the hand and trigeminal levels, there was a significant increase of N2-P2 complex and P2 wave amplitudes in the pre-menstrual phase (Figs. 1, 2; Table 1).

\section{Pain rating}

The laser pain, measured by $0-100$ VAS, was also increased in the pre-menstrual phase in both migraine and control groups (Fig. 3; Table 1).

\section{N2-P2 habituation}

Considering the mean N2-P2 percent ratio of amplitude variation between the third and the first repetition, computed across the two phases, a reduced habituation was present in migraine patients at both the hand (two-ways ANOVA with group as factor $F=4.9$, df $1, P 0.039$ ) and the face levels (two-ways ANOVA with group as factor $F=5.63$, df 1, $P$ 0.027). Moreover, this ratio was further reduced in both groups for the effect of phase at the hand (two-ways ANOVA with phase as factor $F=4.95$, df 1 , $P 0.035$; phase $\times$ group $F=0.012, P 0.78)$ and the supraorbital zone level (phase as factor $=11.75, P 0.0025$, phase $\times$ group $F=1.2, P$ 0.47). In fact, the LEP vertex complex habituation decreased in the premenstrual phase in both patients and controls (Fig. 4).
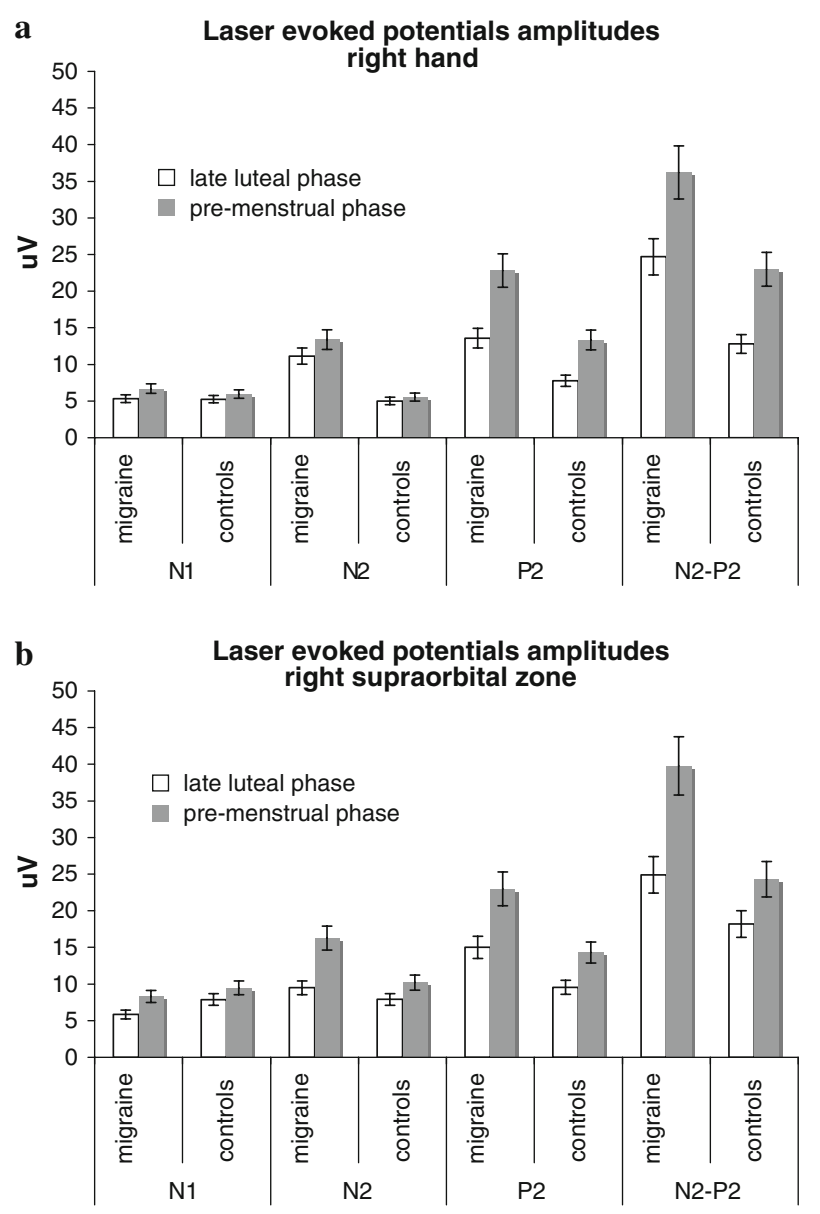

Fig. 1 Mean values and standard errors of laser evoked potentials amplitude in migraine patients (no. 9) and controls (no. 10)

\section{Discussion}

In this study we firstly confirmed previous findings about LEPs in migraine. In fact, in migraineurs the average value of N2-P2 amplitude habituation, computed across the two phases, was reduced at both the hand and trigeminal level $[18,20]$. According to previous studies where repetitive laser stimulations series were considered in patients and controls [20], a global increase of the averaged LEPs concurred with the phenomenon of reduced habituation.

Moreover, this study provided the first objective neurophysiologic evidence about modifications of the brain responses to painful stimuli across the menstrual phases in normal and migraine women. Despite the low number of enrolled subjects, an increase of pain perception emerged during the pre-menstrual phase, as compared to the late luteal phase, at both the hand and trigeminal levels, independently from migraine diagnosis, confirming previous results [12].

In line with the subjective pain rating, the cycle phase modified also the LEP amplitudes in migraineurs and 
Table 1 Results of two way ANOVA applied to laser evoked potentials amplitudes and laser pain, considering the diagnosis, the menstrual phase and the interaction between diagnosis and menstrual phases as the main factors

\begin{tabular}{|c|c|c|c|c|c|c|}
\hline & \multicolumn{3}{|l|}{ Hand } & \multicolumn{3}{|c|}{ Supraorbital zone } \\
\hline & Diagnosis & Menstrual phase & Diagnosis $\times$ phase & Diagnosis & Menstrual phase & Diagnosis $\times$ phase \\
\hline \multicolumn{7}{|c|}{ N1 } \\
\hline$F$ & 0.17 & 1.12 & 0.11 & 0.85 & 1.34 & 0.06 \\
\hline df & 1 & 1 & 1 & 1 & 1 & 1 \\
\hline$P$ & 0.6 & 0.29 & 0.7 & 0.36 & 0.25 & 0.8 \\
\hline \multicolumn{7}{|l|}{$\mathrm{N} 2$} \\
\hline$F$ & 15.78 & 0.63 & 0.085 & 1 & 1.34 & 1 \\
\hline df & 1 & 1 & 1 & 1 & 1 & 1 \\
\hline$P$ & 0.0001 & 0.43 & 0.77 & 0.3 & 0.25 & 0.3 \\
\hline \multicolumn{7}{|l|}{$\mathrm{P} 2$} \\
\hline$F$ & 18 & 10.32 & 1.87 & 8.99 & 5.8 & 0.09 \\
\hline $\mathrm{df}$ & 1 & 1 & 1 & 1 & 1 & 1 \\
\hline$P$ & 0.0001 & 0.003 & 0.18 & 0.005 & 0.022 & 0.7 \\
\hline \multicolumn{7}{|c|}{ N2-P2 } \\
\hline$F$ & 30.80 & 7.9 & 1.68 & 5.32 & 5.22 & 1.55 \\
\hline $\mathrm{df}$ & 1 & 1 & 1 & 1 & 1 & 1 \\
\hline$P$ & 0.0001 & 0.008 & 0.2 & 0.027 & 0.029 & 0.22 \\
\hline \multicolumn{7}{|c|}{ VAS } \\
\hline$F$ & 2.47 & 22 & 2.48 & 1.13 & 6.84 & 0.03 \\
\hline df & 1 & 1 & 1 & 1 & 1 & 1 \\
\hline$P$ & 0.25 & 0.0001 & 0.25 & 0.29 & 0.013 & 0.85 \\
\hline
\end{tabular}

Fig. 2 Grand average of laser evoked potentials across patients and control, obtained by the average of the three consecutive series of laser stimulation at $7.5 \mathrm{~W}$ intensity and $25 \mathrm{~ms}$ stimuli duration. In black: LEPs in the premenstrual phase. In gray: LEPs in the late luteal phase

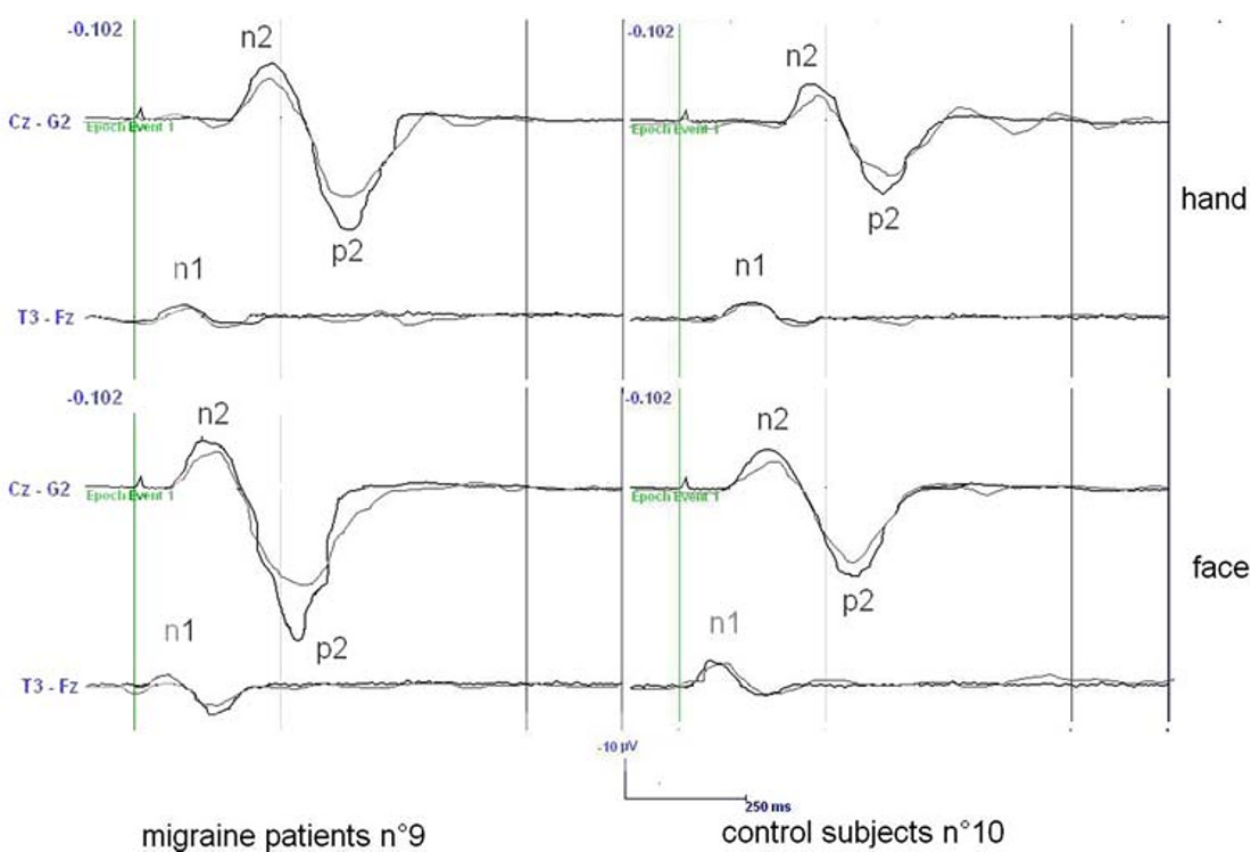

fluctuations occurring during the pre-menstrual phase may facilitate cortical responses to painful stimuli in both women groups, thus showing a pro-nociceptive effect at controls. In fact, in both groups, the P2 wave increment caused an amplitude increase of the N2-P2 complex in the pre-menstrual phase. We can assume that the hormonal 
Subjective perception of laser induced pain, measured by 0-100 VAS

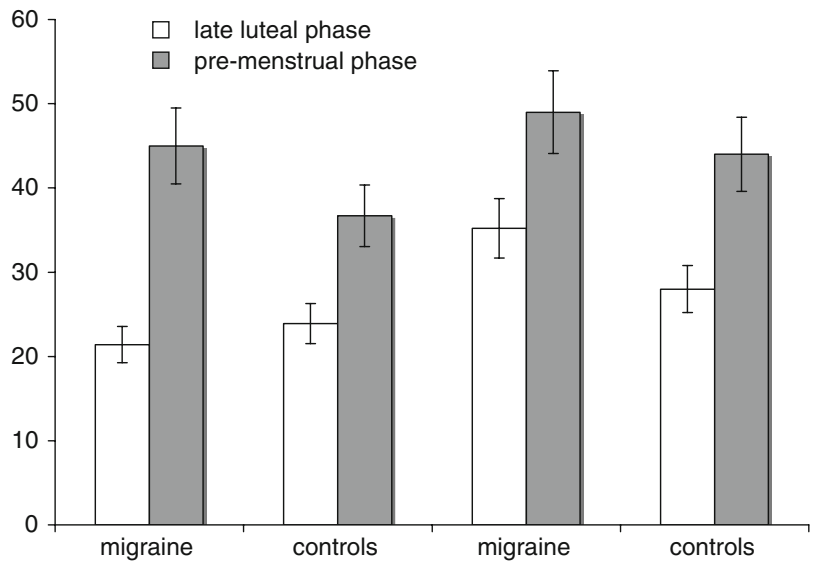

Fig. 3 Mean values and standard errors of VAS values reporting the subjective pain sensation by laser stimuli in migraine patients (no. 9) and controls (no. 10)

percent ratio of $\mathrm{N} 2-\mathrm{P} 2$ amplitude variation $\left(3^{\circ} / 1^{\circ}\right.$ repetition)



Fig. 4 Mean values and standard errors of percent ratio of amplitude variation, computed between the third and the first series of N2-P2 complexes, in migraine patients (no. 9) and controls (no. 10)

central level. The lack of a significant N1 and N2 amplitude increment during the pre-menstrual phase in both patients and controls may be caused by the low number of cases, and deserves further evaluation, in order to confirm the impression that the hormonal influence on the cortical zones devoted to the pain processing is not homogenous. Though our recording method did not enable a topographic and dipolar source analysis of the LEP responses, the later vertex complex changes, which seems to take origin from the anterior cingulate cortex (ACC) [26], may confirm functional magnetic resonance imaging (fMRI) findings, showing an increased activity in the anterior part of the anterior cingulate during the low-estrogen phase of the cycle [27].
The subjective increase of pain rating, concurring with enhanced activity of cortical areas devoted to noxious stimuli elaboration, was independent of migraine diagnosis, and was present at the hand and trigeminal levels. The correlation between pain in the head and menstrual cycle has been recognized since Hippocrates [5] and may be a direct effect of this phenomenon. It has been recently suggested that also tension-type headache is more frequent during the menstrual phase, so that the menstrual-related tension-type headache may be a clinical entity [28].

In control women, the N2-P2 amplitude habituation decreased during the pre-menstrual phase, as well as in migraine patients. In migraine, the pattern of reduced habituation to repetitive stimuli has been generally attributed to an abnormal cortical excitability [2]. In control women, the neuro-transmission modifications linked to estrogen and progesterone withdrawal [29] may cause changes of the brain excitability that could become more similar to the migraineur one. This hypothesis may also explain the increased probability to develop a migraine attack in the pre-menstrual phase in women with genetic predisposition to migraine. Subjects with familiar risk of migraine, showed reduced habituation of nociceptive blink reflex as a sign of their susceptibility to develop migraine under precipitating factors [30]. In migraine, high estrogen levels may balance the abnormal neuronal excitability [31]. It has been previously suggested that, when estrogen levels fall at menses, a mismatch in homeostatic gene regulation by estrogen unmasks non-nuclear mitogen-activated hyperexcitability of cell membranes, sensitizing neurons to triggers that activate migraine attacks [31]. A previous study on the CNV, an event related potential whose amplitude and habituation abnormalities have been correlated with cortical functional changes predisposing to migraine [10], showed that during the pre-menstrual phase both migraine patients and non migraine healthy women exhibited a CNV amplitude increase [11]. The increased amplitude and reduced habituation of LEPs in both migraine and non migraine groups, occurring in the premenstrual phase, may be due to a generic hormonal effect on cortical excitability, not specific for nociceptive cortex. Nevertheless, the increased activation of cortical areas devoted to pain elaboration may be critical for migraine attack occurrence [21].

The main limitations of the present study are represented by: (1) the low number of subjects, and (2) the lack of an objective measure of the hormonal levels in the follicular and late lutenic phases.

Therefore, further studies are needed to confirm that the estrogen withdrawal occurring during the menstrual cycle may favor pro-nociceptive changes at cortical level. We can suppose that this may be a factor precipitating pain symptoms and migraine in predisposed women. 
Conflict of interest None.

\section{References}

1. Craft RM (2007) Modulation of pain by oestrogen. Pain 132(suppl 1):S3-S12

2. Goadsby PJ (2005) Can we develop neurally acting drugs for the treatment of migraine? Nat Rev Drug Discov 4(9):741-750

3. Welch KMA (2005) Brain hyperexcitability. The basis for antiepileptic drugs in migraine prevention. Headache 45:S25-S32

4. Welch KMA (2003) Contemporary concepts of migraine pathogenesis. Neurology 61:S2-S8

5. MacGregor AE (2004) Oestrogen and attacks of migraine with and without aura. Lancet Neurol 3:354-361

6. Somerville BW (1972) The role of estradiol withdrawal in the etiology of menstrual migraine. Neurology 22:355-365

7. Brandes JL (2006) The influence of estrogen on migraine: a systematic review. JAMA 295(15):1824-1830

8. Somerville BW (1975) Estrogen-withdrawal migraine, I: duration of exposure required and attempted prophylaxis by premenstrual estrogen administration. Neurology 25:239-244

9. Somerville BW (1975) Estrogen-withdrawal migraine, II: attempted prophylaxis by continuous estradiol administration. Neurology 25:245-250

10. Siniatchkin M, Kropp P, Gerber WD (2001) Contingent negative variation in subjects at risk for migraine without aura. Pain 94: 159-167

11. Siniatchkin M, Averkina N, Gerber WD (2006) Relationship between precipitating agents and neurophysiological abnormalities in migraine. Cephalalgia 26:457-465

12. Stening K, Eriksson O, Wahren L, Berg G, Hammar M, Blomqvist A (2007) Pain sensations to the cold pressor test in normally menstruating women: comparison with men and relation to menstrual phase and serum sex steroid levels. Am J Physiol Regul Integr Comp Physiol 293:R1711-R1716

13. Söderberg K, Sundström Poromaa I, Nyberg S, Bäckström T, Nordh E (2006) Psychophysically determined thresholds for thermal perception and pain perception in healthy women across the menstrual cycle. Clin J Pain 22:610-616

14. Okifuji A, Turk DCJ (2006) Sex hormones and pain in regularly menstruating women with fibromyalgia syndrome. Pain 7:851-859

15. Ambrosini A, de Noordhout AM, Sándor PS, Schoenen J (2003) Electrophysiological studies in migraine: a comprehensive review of their interest and limitations. Cephalalgia 23(suppl 1):13-31

16. Thompson RF, Spencer WA (1996) Habituation: a model phenomenon for the study of neuronal substrates of behavior. Psychol Rev 73(1):16-43

17. de Tommaso M (2008) Laser-evoked potentials in primary headaches and cranial neuralgias. Expert Rev Neurother 8(9): 1339-1345
18. Valeriani M, de Tommaso M, Restuccia D, Le Pera D, Guido M, Iannetti GD, Libro G, Truini A, Di Trapani G, Puca F, Tonali P, Cruccu G (2003) Reduced habituation to experimental pain in migraine patients: a $\mathrm{CO}(2)$ laser evoked potential study. Pain 105(1-2):57-64

19. de Tommaso M, Lo Sito L, Di Fruscolo O, Sardaro M, Pia Prudenzano M, Lamberti P, Livrea P (2005) Lack of habituation of nociceptive evoked responses and pain sensitivity during migraine attack. Clin Neurophysiol 116(6):1254-1264

20. de Tommaso M, Baumgartner U, Sardaro M, Difruscolo O, Serpino C, Treede RD (2008) Effects of distraction versus spatial discrimination on laser-evoked potentials in migraine. Headache 48(3):408-416

21. de Tommaso M, Guido M, Libro G, Losito L, Difruscolo O, Puca F, Specchio LM, Carella A (2004) Topographic and dipolar analysis of laser-evoked potentials during migraine attack. Headache 44(10):947-960

22. Headache Classification Committee (2004) The international classification of headache disorders II. Cephalalgia 24:24-136

23. Biehl R, Treede R-D, Bromm B (1984) Pain ratings of short radiant heat pulses. In: Bromm B (ed) Pain measurement in man. Neurophysiological correlates of pain. Elsevier, Amsterdam, pp 397-408

24. Valeriani M, Rambaud L, Mauguière F (1996) Scalp topography and dipolar source modelling of potentials evoked by $\mathrm{CO}_{2}$ laser stimulation of the hand. Electroencephalogr Clin Neurophysiol 100(4):343-353

25. Cruccu G, Aminoff MJ, Curio G, Guerit JM, Kakigi R, Mauguiere F, Rossini PM, Treede RD, Garcia-Larrea L (2008) Recommendations for the clinical use of somatosensory-evoked potentials. Clin Neurophysiol 119(8):1705-1719

26. Garcia-Larrea L, Frot M, Valeriani M (2003) Brain generators of laser-evoked potentials: from dipoles to functional significance. Neurophysiol Clin 33:279-292

27. de Leeuw R, Albuquerque RJ, Andersen AH, Carlson CR (2006) Influence of estrogen on brain activation during stimulation with painful heat. J Oral Maxillofac Surg 64(2):158-166

28. Arjona A, Rubi-Callejon J, Guardado-Santervas P, Serrano-Castro P, Olivares J (2007) Menstrual tension-type headache: evidence for its existence. Headache 47(1):100-103

29. Martin VT, Behbehani M (2006) Ovarian hormones and migraine headache: understanding mechanisms and pathogenesis. Headache 46:365-386

30. Di Clemente L, Coppola G, Magis D, Fumal A, De Pasqua V, Di Piero V, Schoenen J (2007) Interictal habituation deficit of the nociceptive blink reflex: an endophenotypic marker for presymptomatic migraine? Brain 130:765-770

31. Welch KM, Brandes JL, Berman NE (2006) Mismatch in how oestrogen modulates molecular and neuronal function may explain menstrual migraine. Neurol Sci 27(suppl 2):S190-S192 